\title{
STRUCTURE AND EQUILIBRIUM OF CORONAL MAGNETIC FIELDS
}

\author{
A. A. VAN BALLEGOOIJEN \\ Harvard-Smithsonian Center for Astrophysics \\ 60 Garden Street \\ Cambridge, MA 02138, U.S.A.
}

\begin{abstract}
In "closed" magnetic structures (i.e., coronal loops) the random shuffling of magnetic footpoints in the photosphere causes twisting and braiding of field lines in the corona. If the motions are sufficiently slow, the coronal field evolves through a sequence of force-free equilibrium states. Numerical simulations are presented for a simplified model in which the overall curvature of the coronal loop is neglected. It is shown that magnetic fine structures develop on spatial scales significantly smaller than those of the imposed "photospheric" velocity field.
\end{abstract}

\section{Introduction}

It has been suggested that coronal loops are heated by dissipation of field-aligned electric currents (e.g., Gold 1964; Parker 1972; Tucker 1973; Rosner et al. 1978). These currents arise as a result of footpoint motions driven by subsurface convective flows. Since the magnetic field is nearly "frozen" into the plasma, the random walk of the footpoints across the solar surface causes twisting and braiding of field lines in the corona. If the footpoint motions are slow compared to the Alfven time $L / v_{A}$ and the plasma pressure is negligible compared to the magnetic pressure, then the force balance requires that the Lorentz force nearly vanishes, $\mathbf{j} \times \mathbf{B}=0$; hence, electric currents associated with such twisted fields flow nearly parallel or anti-parallel to the magnetic field lines.

In this paper I present results from a numerical model in which the overall curvature of the coronal loop is neglected: the initial field is assumed to be uniform, extending between two parallel plates which represent the solar photosphere at the two "ends" of the loop. The field is assumed to evolve according to ideal MHD. The formation of current sheets in this model was discussed by Parker $(1972,1983,1986 \mathrm{a}, \mathrm{b})$ and van Ballegooijen $(1985,1986$, 1988a). The latter showed that spatially continuous flows at the boundary plates produce spatially continuous force-free fields inside the volume. Numerical results were presented earlier by van Ballegooijen (1988b).

\section{Numerical Method and Results}

A Cartesian reference frame $(x, y, z)$ is adopted, with the boundary plates located at $z=0$ and $z=L$. The initial field (time $t=0$ ) is given by $\mathbf{B}(x, y, z, 0)=B_{0} \mathbf{z}$. For simplicity I assume

E. R. Priest and V. Krishan (eds.), Basic Plasma Processes on the Sun, 303-308.

(C) 1990 IAU. Printed in the Netherlands. 
that the field lines are fixed at $z=0$, and that the velocity at $z=L$ consists of a series of sinusoidal flow patterns with "wavelength" $\lambda \ll L$. Specifically, the velocity $\mathbf{v}_{n}(x, y, L)$ for times $t$ in the range $(n-1) \Delta t<t<n \Delta t$ (with $n=1,2, \ldots$ ) is given by:

$$
\begin{aligned}
& \mathrm{v}_{n, x}=0, \quad \mathrm{v}_{n, y}=A \sin \left(k x+\phi_{n}\right), \quad \text { for } n \text { odd, } \\
& \mathrm{v}_{n, x}=A \sin \left(k y+\phi_{n}\right), \quad \mathrm{v}_{n, y}=0, \quad \text { for } n \text { even, }
\end{aligned}
$$

where $A$ is the velocity amplitude, $k(=2 \pi / \lambda)$ is the wavenumber, and the $\phi_{n}$ are arbitrary phase angles $\left(v_{z}=0\right)$. The magnetic field is described in terms of the paths of the field lines, $x=X\left(x_{0}, y_{0}, z, t\right)$ and $y=Y\left(x_{0}, y_{0}, z, t\right)$, where $x_{0}$ and $y_{0}$ are the initial coordinates of a field line at time $t=0$. Then the magnetic field is:

$$
\mathbf{B}\left(x_{0}, y_{0}, z, t\right) \approx \frac{B_{0}}{J}\left[\frac{\partial X}{\partial z}, \frac{\partial Y}{\partial z}, 1\right],
$$

where $J\left(x_{0}, y_{0}, z, t\right)$ is the Jacobian of the transformation $\left(x_{0}, y_{0}\right) \rightarrow(X, Y)$. The functions $X$ and $Y$ satisfy the following boundary conditions:

$$
\begin{array}{lc}
X\left(x_{0}, y_{0}, 0, t\right)=x_{0}, & Y\left(x_{0}, y_{0}, 0, t\right)=y_{0}, \\
X\left(x_{0}, y_{0}, L, t\right)=X_{t o p}\left(x_{0}, y_{0}, t\right), & Y\left(x_{0}, y_{0}, L, t\right)=Y_{t o p}\left(x_{0}, y_{0}, t\right),
\end{array}
$$

where $X_{\text {top }}$ and $Y_{\text {top }}$ are the positions of the footpoints at $z=L$, as determined from equations (1a) and (1b). In the limit $\lambda \ll L$ the magnetic free energy $\Delta W$ of the system is given by

$$
\left.\Delta W \approx \frac{B_{0}^{2}}{8 \pi} \int_{0}^{L \lambda \lambda} \int\left[\int \frac{\partial X}{\partial z}\right]^{2}+\left[\frac{\partial Y}{\partial z}\right]^{2}+(J-1)^{2}\right] d x_{0} d y_{0} d z
$$

By minimizing $\Delta W$ subject to the constraints (3a,b), a force-free magnetic field is obtained (Sakurai 1979). I introduce a Lagrangian grid tied to the field lines. Let $X_{i j k}$ and $Y_{i j k}$ denote the values of $X\left(x_{0}, y_{0}, z\right)$ and $Y\left(x_{0}, y_{0}, z\right)$ at a grid point. Replacing the partial derivatives in equation (4) by their finite-difference analogs and the integrations by sums, the magnetic energy $\Delta W$ may be written as a function of the variables $X_{i j k}$ and $Y_{i j k}$ at all interior grid points. I determine the minimum of this function using the conjugate-gradient method (Press et al. 1986). This is a general-purpose method for finding the minimum of a function of a large number of variables.

Computations were made for nine sets of randomly chosen phase angles. A velocity amplitude $A k \Delta t=1$ was assumed. Calculations for time steps $n \leq 4$ were made with a grid of $64 \times 64 \times 8$ points (including the points on the boundaries), while calculations for $n=5$ were made with a grid of $128 \times 128 \times 11$ points. For $n>5$ not all nine cases could be adequately resolved, even with the larger grid. Plots of the projections of field lines onto a plane $z=$ constant show that the field lines are more or less straight lines connecting the footpoints. This indicates a tendency of the field lines to be as short as possible. Contour plots of the electric current density at $z=0$ were also produced. As time progresses, the electric currents become stronger and elongated regions with high current density develop ("current sheets"). Examples of such plots are given by van Ballegooijen (1988b) and by Mikic et al. (1989). Figure 1 shows contour plots of the average power spectrum of the current density. This clearly shows the progression of power towards higher wavenumbers, indicative of the development of small-scale structure in the electric-current distribution. 

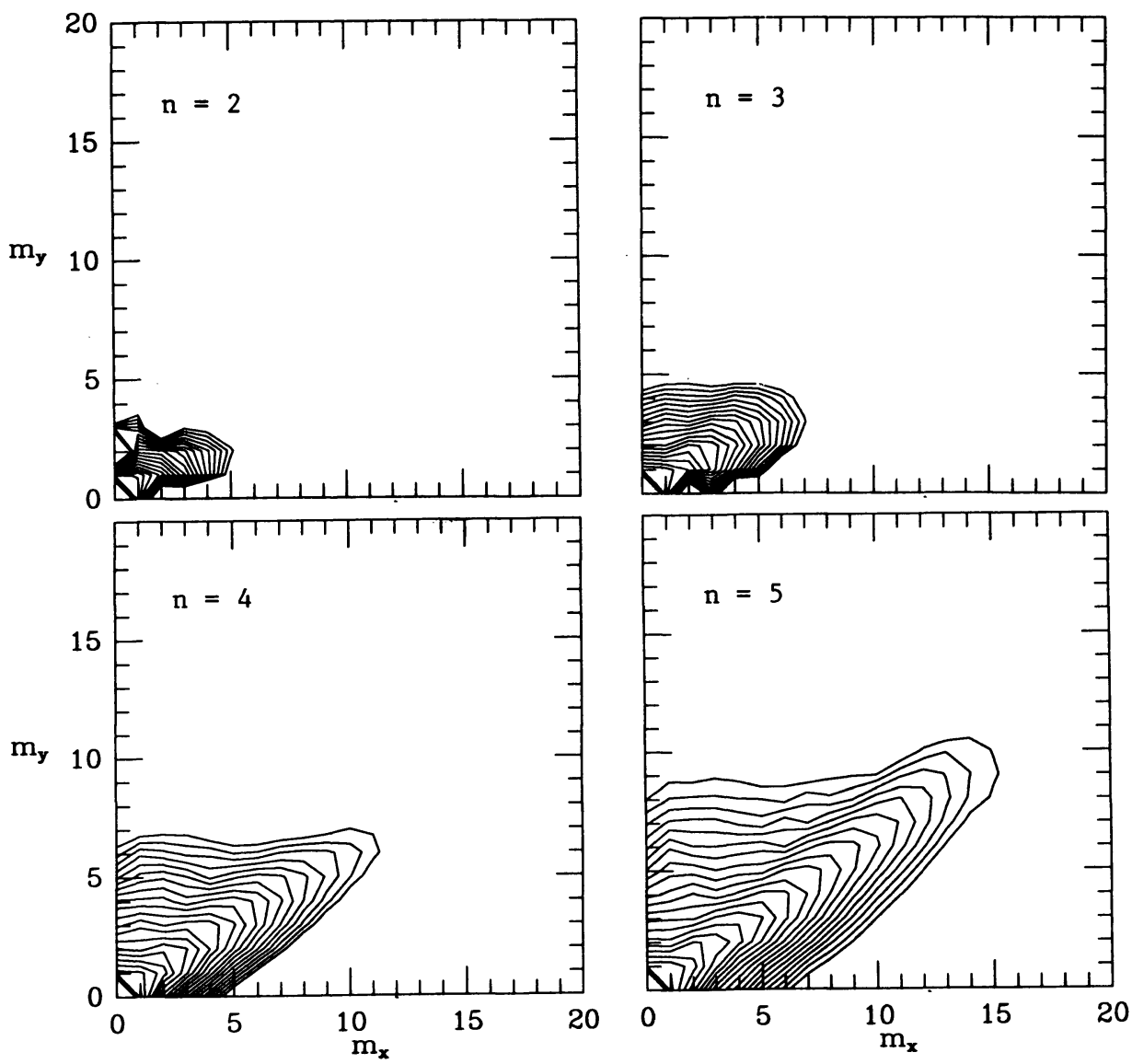

Figure 1. Contour plots of the spatial power spectrum of current-density fluctuations as measured at $z=0$, for different times $n$. The average over nine realizations of random phase angles is shown. Neighboring contours are spaced by a factor 2 in power.

The fact that field lines do not deviate strongly from straight lines suggests the following linear approximation:

$$
\begin{aligned}
& X\left(x_{0}, y_{0}, z, t\right)=x_{0}+\left[X_{t o p}\left(x_{0}, y_{0}, t\right)-x_{0}\right] z / L, \\
& Y\left(x_{0}, y_{0}, z, t\right)=y_{0}+\left[Y_{t o p}\left(x_{0}, y_{0}, t\right)-y_{0}\right] z / L .
\end{aligned}
$$

Although this does not generally represent a physically possible magnetic field ( $J$ may be negative), it does provide a lower bound on the magnetic free energy of the force-free field. Also, it provides the correct solution in the limit of small displacements, i.e., when $\left|X_{\text {top }}-x_{0}\right|$ and $\left|Y_{\text {top }}-y_{0}\right|$ are small compared to $\lambda$ (see Zweibel and Li 1987). Inserting (5a,b) into the first two terms of equation (4), and neglecting the third term, I obtain the following expression for the lower bound:

$$
\Delta W_{\min }=\frac{B_{0}^{2}}{8 \pi L} \int_{\delta}^{\lambda \lambda}\left\{\left[X_{t o p}\left(x_{0}, y_{0}, t\right)-x_{0}\right]^{2}+\left[Y_{t o p}\left(x_{0}, y_{0}, t\right)-y_{0}\right]^{2}\right\} d x_{0} d y_{0}
$$


I find that with $A k \Delta t=1$ the actual energy $\Delta W$ does not exceed $\Delta W_{\min }$ by more than about $15 \%$. Also, the distributions of electric current at $z=0$ derived from expressions $(5 a, b)$ are very similar to the computed, force-free distributions. Thus, we have not yet reached the regime where the field lines are strongly braided. Apparently, strong braiding implies the existence of even smaller spatial scales that cannot yet be resolved.

\section{Conclusion}

The simulations show that random motions of footpoints can produce fine structures in the electric-current distribution on scales significantly smaller than $\lambda$, the scale of the imposed velocity field. However, the field lines are still relatively simple; they can be crudely approximated as straight lines. The numerical resolution is not large enough to simulate strongly braided magnetic fields, with circuitous paths of the field lines. Further improvements in numerical methods are required.

\section{Acknowledgement}

This work was supported by NASA under grants NAGW-249 and NAGW-1568.

\section{References}

Gold, T. 1964, in The Physics of Solar Flares, ed. W. Hess (NASA SP-50), p. 389

Mikic, Z., Schnack, D.D., and Van Hoven, G. 1989, Ap. J. 338, 1148

Parker, E.N. 1972, Ap. J. 174, 499

----- 1983, Ap. J. 264, 642

1986a, Geophys. Astrophys. Fluid Dyn. 34, 243

1986b, Geophys. Astrophys. Fluid Dyn. 35, 277

Press, W.H., Flannery, B.P., Teukolsky, S.A., and Vetterling, W.T. 1986, Numerical Recipes (Cambridge University Press), Chap. 10.6

Rosner, R., Golub, L., Coppi, B., and Vaiana, G.S. 1978, Ap. J. 222, 317

Sakurai, T. 1979, Pub. Astron. Soc. Japan 31, 209

Tucker, W. 1973, Ap. J. 186, 285

van Ballegooijen, A.A. 1985, Ap. J. 298, 421

1986, Ap. J. 311, 1001

-.--.-1 1988a, Geophys. Astrophys. Fluid Dyn. 41, 181 spot, NM: National Solar Observatory), p. 115

Zweibel, E.G., and Li, H. 1987, Ap. J. 312, 423 


\section{DISCUSSION}

MONTGOMERY: Do you have a velocity field away from the end plate? If so, how is it advanced in $\mathrm{z}$ and $\mathrm{t}$ ? Physically, one would think that forcing the velocity field to have a definite temporal behaviour at the end plate would launch Alfven waves, and that is where much of the energy might go.

VAN BALLEGOOIJEN: Yes, there is a velocity field away from the end plates, but it is not explicitly considered in the code. The force-free problem is treated as a boundary-value problem; the advance in $t$ of $(x, y)$ positions is done only at the boundary plates. If the transverse velocity is small compared to the Alfvén speed and the velocity varies on a timescale $(\Delta t)$ long compared to $L / V_{A}$, significant Alfvén waves are probably not launched; the field evolves quasi-statically.

MOGILEVSKIJ: What is the energy density of the force-free magnetic fields?

VAN BALLEGOOIJEN: The magnetic free energy is at most a few percent of the potential-field energy; it scales with $(\lambda / L)^{2}$.

DRYER: (i) In order to achieve the large amount of braiding, do you think it may be necessary to go to an initial-boundary value problem with the presence of pressure, velocity field, etc.?

(ii) Would you comment on the conclusion of Klimchuk and Sturrock (Ap.J., Oct 1989) that loss-of-equilibria, quasi-static, force-free calculations have no physical basis unless the plasma pressure and gravity are included?

VAN BALLEGOOIJEN: (i) Gas pressure is not essential, but it might be numerically advantageous to use an initial-value approach as Mikic et al. have done.

(ii) Loss-of-equilibrium may occur in force-free fields if 3D effects are taken into account. They may occur in the context of the Parker problem, but I have not found any evidence for non-equilibrium in my calculation, perhaps because the free energy is much smaller than the potential-field energy.

PRIEST: (i) What does $\mathbf{t}=\mathbf{5}$ in your nondimensionalisation mean. How many time steps would you need to produce braiding?

(ii) Is it a problem of numerical stability or computer storage which stops you going beyond $\mathrm{t}=5$ ?

(iii) How do your results differ from those of Mikic?

(iv) How long would it take for the current densities to build up to values where magnetic dissipation is important?

VAN BALLEGOOIJEN: (i) Each velocity pattern persists for a time $\Delta t$; " $t=5$ " refers to the magnetic field at time $t=5 \Delta$. Braiding probably requires several tens of $\Delta t$.

(ii) Numerical errors develop for $\mathrm{t}=6 \Delta \mathrm{t}$ for some sets of phase angles. I would not call it a problem of "numerical stability" because the energy-minimization scheme always finds a lowest energy state.

(iii) The results are nearly identical to those of Mikic et al. when I use the same phase angles. However, they did not do the averaging over different sets of phase angles.

(iv) Several tens of $\Delta t$. 
HOLLWEG: A key issue in the field-stressing models is how much energy flux you can in fact launch into the corona. For realistic numbers, do you have a feel yet for how much flux you can launch into a loop? I still have the impression that one has to stretch the numbers to get the required energy fluxes.

VAN BALLEGOOIJEN: Earlier analytical estimates (Van Ballegooijen 1986) suggested a heating rate per unit volume $\varepsilon \simeq 0.2 \mathrm{~B}^{2} \mathrm{D} / \mathrm{L}^{2}$, where $\mathrm{D}$ is the "diffusion constant" of photospheric motions. Taking $\mathrm{D} \sim 400 \mathrm{~km}^{2} \mathrm{~s}^{-1}$ (De Vore et al. 1985), the heating rate is a factor $\mathbf{4 0}$ too small, but there are many uncertainties in these estimates, and so we should not rule out DC currents as a mechanism for coronal heating.

DAVILA: In your present numerical calculation are you near the statistical steady state where the energy input at the boundary is balanced by current dissipation within the interior of the model?

VAN BALLEGOOIJEN: No, on the contrary, these calculations show only the initial development of the cascade. Magnetic diffusion (which is neglected here) becomes important only much later, when very thin current layers have developed.

KUIJPERS: The coronal field is anchored in discrete subphotospheric elements. I would expect the velocity field not to vary much over an individual magnetic element. Therefore, are your calculations applicable primarily to the boundaries between individual flux tubes rooted in separate magnetic elements?

VAN BALLEGOOIJEN: The sinusoidal velocity patterns used in these calculations are indeed not very realistic and do not take into account the flux-tube nature of the photospheric field. Indeed, if there are discrete flux tubes, magnetic discontinuities develop at the interfaces between these flux tubes in the corona. Hence, current sheets probably develop even more rapidly than suggested here.

NARAIN: How do you differ from Parker? What is your estimate for heating solar corona?

VAN BALLEGOOIJEN: Parker proposes that, in ideal MHD, current sheets form "spontaneously" (i.e., sheet thickness vanishes at finite time), whereas I propose that current sheets develop "gradually" (sheet thickness decreases exponentially with time). I agree, however, with Parker that current dissipation is a promising mechanism for coronal heating. 